\title{
Article \\ Improved Enzymatic Assay and Inhibition Analysis of Redox Membranotropic Enzymes, AtGALDH and TcGAL, Using a Reversed Micellar System
}

\author{
Andrey A. Chudin and Elena V. Kudryashova *
}

check for

updates

Citation: Chudin, A.A.;

Kudryashova, E.V. Improved

Enzymatic Assay and Inhibition

Analysis of Redox Membranotropic

Enzymes, AtGALDH and TcGAL,

Using a Reversed Micellar System.

Analytica 2022, 3, 36-53. https:/ /

doi.org/10.3390/analytica3010004

Academic Editors: Marcello Locatelli,

Victoria Samanidou, Roberto

Mandrioli and Thomas W. Bocklitz

Received: 28 December 2021

Accepted: 27 January 2022

Published: 3 February 2022

Publisher's Note: MDPI stays neutral with regard to jurisdictional claims in published maps and institutional affiliations.

Copyright: (C) 2022 by the authors. Licensee MDPI, Basel, Switzerland. This article is an open access article distributed under the terms and conditions of the Creative Commons Attribution (CC BY) license (https:/ / creativecommons.org/licenses/by/ $4.0 /)$.
Faculty of Chemistry, Lomonosov Moscow State University, Leninskie Gory 1/3, 119991 Moscow, Russia; andrew_18@inbox.ru

* Correspondence: helenakoudriachova@yandex.ru

\begin{abstract}
Reversed micelles are helpful to solubilize otherwise insoluble membranotropic or membranebound enzymes in their functional form, thus enabling activity assay and inhibition analysis. However, in the case of redox enzymes, this task is further complicated by the necessity to select an appropriate electron-acceptor (EA) which, ideally, should be compatible with spectrophotometric measurements in reversed micelles. Here, we have identified such an EA and successfully used it in a reversed micellar environment to assay the activity of two homologous enzymes from mitochondria: L-galactone-1,4-lactone dehydrogenase (EC 1.3.2.3) from Arabidopsis thaliana (AtGALDH) and galactonolactone oxidase (EC 1.3.3.12) from Trypanosoma cruzi (TcGAL), differing in their membranotropic properties, with TcGAL being almost insoluble in water and particularly difficult to assay. Furthermore, we have demonstrated the possibility to use this assay for inhibition analysis, with an elucidation of the mechanism and inhibition parameters, which otherwise could not be possible. In order to perform inhibition analysis, we improved the approach for the determination of activity of such membrane enzymes based on a reversed micellar system as membrane matrix, necessary for the functioning of membrane enzymes. A number of electron acceptors (EA) were tested for AtGALDH and optimal conditions of activity determination for AtGALDH were found. The suggested method was successfully applied to the study of the inhibition of AtGALDH by lycorine, and the mixed competitive mechanism of inhibition of AtGALDH by lycorine was determined. The developed approach to inhibitor analysis was applied for TcGAL, insoluble in water membrane, and the method provides new opportunities for searching effective inhibitors that may be potential drugs. Indeed, galactonolactone oxidase from Trypanosoma cruzi (TcGAL) and AtGALDH are homologues, and the inhibition of TcGAL stops the vital biosynthesis of vitamin C in parasite Trypanosoma cruzi from causing Chagas disease. The approach proposed can be applied for the screening of inhibitors of AtGALDH and TcGAL, as well as to study properties of other membrane enzymes including determination of the mechanisms of inhibition, structure and catalytic properties, the impact of membrane components (for example lipids), and so on.
\end{abstract}

Keywords: determination of membrane enzyme activity; spectrophotometric method; membrane enzymes; L-galactone-1,4-lactone dehydrogenase; Arabidopsis thaliana; galactonolactone oxidase; Trypanosoma cruzi; lycorine; drugs; Chagas disease

\section{Introduction}

The intensive development of structural biology in the past few years has made it possible to determine the structures of many integral membrane enzymes. Membrane enzymes plays an important role in living organisms, and studying their properties is necessary for a detailed understanding of metabolism pathways, regulation mechanisms and other aspects of life. However, the studies of membrane enzymes are complicated, since many of these enzymes are insoluble in water, and there are no effective methods of determination of their activity. Recent research has demonstrated the similarity between 
active site composition of insoluble membrane enzymes and water-soluble ones catalyzing the same types of reactions [1], which allows the use of soluble proteins as models (these enzymes are also membranotropic). Nevertheless, due to the complex physicochemical organization of cellular membranes, as well as the presence of transmembrane gradients and potentials, these enzymes may be subject to membrane-specific regulatory mechanisms, the study of which is just beginning.

One of the most promising areas related to membrane enzymes is the development of drugs based on inhibitors that suppress the biosynthesis of vital compounds in pathogenic parasites. One of these parasites is Trypanosoma cruzi causing Chagas disease, which is nowadays treated with only two drugs: benznidazole and nifurtimox. However, these drugs have significant disadvantages, including their efficacy only in the early or acute infection phases, side effects, and the resistance that the parasite has developed to their activity [2]. To fully understand the scale of the problem it should be added that about 7 million people all over the world suffer from Chagas disease and, moreover, the disease has recently spread in Europe, the United States and Canada [3]. Thus, the development of new drugs against Chagas disease is an significant task.

As mentioned above, the action of such drugs may be based on their inhibitory effect on the biosynthesis of compounds vital for the parasite. For example, it is known that $T$. cruzi is completely dependent on endogenously produced sterols necessary for its survival. Thus, the enzymes involved in the ergosterol biosynthesis pathway in the T. cruzi parasite are considered as an attractive target for drug development [2,4].

Another promising approach is the inhibition of the final step of the synthesis of ascorbate, a vital compound that the parasite cannot take up from its environment, relying on de novo synthesis for its survival [5]. Galactonolactone oxidase (EC 1.3.3.12) from T. cruzi (TcGAL), catalyzing this step (Scheme 1), belongs to water-insoluble membrane enzymes, so to study this protein, one has to use a lipid membrane model system (reverse micelles) and refolding.<smiles>CC1(C)C2CCCC1C2OCC1OC(=O)C(O)=C1O</smiles>

Scheme 1. The reaction catalyzed by Galactonolactone oxidase from T. cruzi (TcGAL).

However, the preliminary screening of TcGAL potential inhibitors can be performed using the homologues model enzyme L-galactone-1,4-lactone dehydrogenase (EC 1.3.2.3) from Arabidopsis thaliana (AtGALDH), which is active in both aqueous and model environments, does not require refolding, and shows $21 \%$ sequence identity to TcGAL [5]. The reaction catalyzed by AtGALDH is presented in Scheme 2.
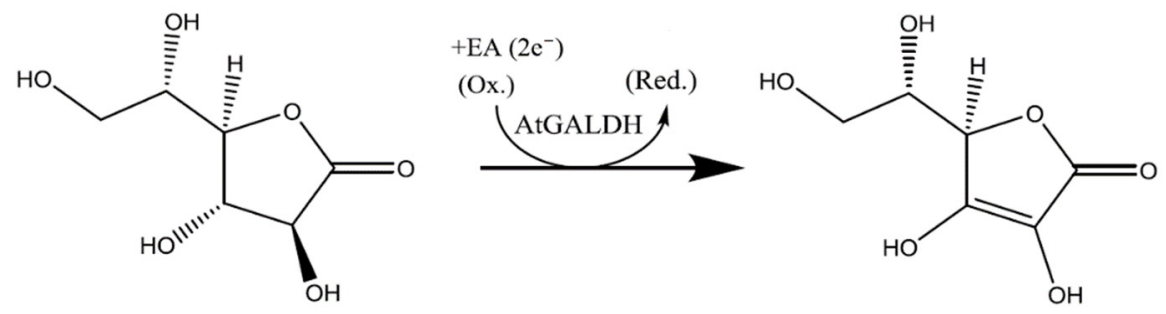

Scheme 2. The reaction catalyzed by L-galactone-1,4-lactone dehydrogenase from Arabidopsis thaliana (AtGALDH).

Many inhibitors of AtGALDH and L-galactone-1,4-lactone dehydrogenases from other sources have either low specificity (for example, 5,5'-dithiobis(2-nitrobenzoic acid) which acts by the oxidation mechanism of Cys-340 of AtGALDH [6]), or high toxicity (mercury- 
containing compounds, such as p-chloromercuribenzoate which inhibits L-galactone-1,4lactone dehydrogenase from Nicotiana tabacum [7]). Lycorine, an alkaloid found in various Amaryllidaceae species, has anti-inflammatory, antiviral, antibacterial and many other pharmacological effects [8], it has long been used as a medicine and is devoid of the above disadvantages. Lycorine (the structure is presented in Figure 1) is known as a strong inhibitor of the number of L-galactone-1,4,-dehydrogenases from different sources, such as sweet potato (Ipomoea batatas) [9], or Zea mays seedlings [10] (90-100\% inhibition at a concentration of $10-100 \mu \mathrm{M}$ of lycorine). However, there are no publications on the effect of lycorine on the activity of the TcGAL or AtGALDH.<smiles>O[C@H]1C=C2CCN3Cc4cc5c(cc4[C@H]2[C@@H](O)[C@@H]13)OCO5</smiles>

Figure 1. The structural formula of lycorine.

A relatively robust method for measuring AtGALDH activity in aqueous media is the spectrophotometric method, following the reduction of natural electron acceptors cytochrome $C$ at $550 \mathrm{~nm}$, as described by the authors in [11]. However, this method is not suitable for reverse micelles systems (which are used as membrane models for the investigation of water-insoluble membrane enzymes) because of a strong interaction of cytochrome $C$ with the surfactant interface [12], which leads to a loss of cytochrome $\mathrm{C}$ secondary structure [13]. Thus, given that AtGALDH and TcGAL are two-substrate enzymes (one substrate is L-galactone-1,4-lactone or D-arabinono-1,4-lactone, the other is an electron acceptor (EA)), the study and selection of new, more effective EAs may improve the spectrophotometric determination of the enzyme's activity (as well as optimization with already studied EAs).

The micellar approach mentioned above is a powerful instrument for the study of membrane enzymes, and thus should be discussed in detail. The influence of lipids in living cells should be taken into account, since lipids bind specific domains on the protein surface, change the microviscosity and fluidity of the lipid bilayer, and thus strongly affect the functions of membranotropic enzymes. The reverse micelles system is suggested as a direct tool to study the influence of membrane matrix composition on the activity and structure of membrane-associated enzymes [14]. The sizes of the inner cavities of the micelles where protein molecules are entrapped can be strictly controlled by varying the surfactant hydration degree $\left(\mathrm{W}_{0}\right)$, which is determined by the molar ratio of the water surfactant [15]. The catalytic activity of enzymes in reverse micelles usually shows a "bellshaped" dependency on the $\mathrm{W}_{0}$ value [14]. Maximum activity is observed at the hydration degree, $W_{0}$, where the size of a micelle is equal to that of the entrapped protein. In the case of oligomeric enzymes, the dependence of kinetic parameters on $W_{0}$ shows peaks corresponding to each oligomeric form of an enzyme [16].

It should be underlined that reverse micelles are rather realistic membrane structure models. It has been found that bilayer membranes often contain non-bilayer structures, so-called lipid particles, which are clearly visible in electron microscopic images. Studies have shown that these particles are associates of lipid molecules, built like reverse micelles and enclosed between monolayers of a bilayer membrane [17].

In addition, purification of some soluble enzymes results in a considerable loss of enzyme activity. The problem can be solved by obtaining them from the insoluble inclusion bodies fraction with the use of the micellar approach. Here, TcGAL was obtained from inclusion of bodies in active form using the water-AOT-isooctane micellar system and 
TcGAL and the new developed system of analysis of TcGAL activity with use of the most effective electron acceptor was applied.

Nevertheless, there is still no micellar approach to the inhibition analysis of membrane enzymes, and there are no effective methods for the determination of the activity of these enzymes. Thus, the aim of this work is to improve the micellar approach to inhibition analysis of membrane enzymes, with AtGALDH and TcGAL as examples.

\section{Materials and Methods}

\subsection{Chemicals}

L-galactono-1,4-lactone, D-arabinono-1,4-lactone, bovine heart cytochrome C, 2,6dichlorophenolindophenol (DCPIP), bis(2-ethylhexyl)sulfosuccinate (AOT), n-octane, acetonitrile, sodium borohydride were from Sigma-Aldrich (St Louis, MO, USA), phenazine methosulfate and tetramethylphenylenediamine dihydrochloride were from Merck (Darmstadt, Germany), lycorine hydrochloride was from Aladdin (Shanghai, China).

E. coli strain BL21(DE3) was from Novagen (San Diego, CA, USA). Isopropyl thio$\beta$-d-galactoside (IPTG) was obtained from MP Biomedicals (Irvine, CA, USA). Nickel nitrilotriacetic acid (Ni-NTA) agarose was purchased from Qiagen (Valencia, CA, USA). HiLoad 2610 Q-Sepharose HP was obtained from Pharmacia Biotech (Uppsala, Sweden).

The $\mathrm{p}$ TrcHisC vector containing the TcGAL gene (from T. cruzi X10/6 clone) N-terminally fused to a $\mathrm{His}_{6}$-tag, XpressTM epitope and enterokinase cleavage site, was kindly provided by Prof.M.W. Fraaije (University of Groningen). Restriction endonucleases, T4-DNA ligase and dNTPs were purchased from Invitrogen (Carlsbad, CA, USA). Pfu DNA polymerase was obtained from Fermentas $\mathrm{GmbH}$ (St Leon-Rot, Germany). Oligonucleotides were synthesized by Eurogentec (Liege, Belgium). All other chemicals were from commercial sources and of the purest grade available.

\subsection{Instrumentation}

Kinetic measurements (determination of the enzyme activity) and registration of spectra were performed on an Ultrospec-2100 pro spectrophotometer "Amersham biosciences" (Pittsburgh, PA, USA) with a thermostated cuvette compartment (all measurements were carried out at room temperature); data were recorded and processed using the Datalyse 3.70 program. French Pressure cell was from SLM Aminco, SLM Instruments, Urbana, IL, USA. SigmaPlot 11.1 software was used to plot the graphs. Catalytic parameters of systems were calculated using the non-linear regression method. Sequence alignments were performed using the Clustal Omega program (with the use of UniProt database).

\subsection{Recombinant Expression of AtGALDH and TcGAL}

Recombinant enzyme preparations: L-galactone-1,4-lactone dehydrogenase GALDH (EC 1.3.2.3) from Arabidopsis thaliana (AtGALDH) and galactonolactone oxidase (EC 1.3.3.12) from Trypanosoma cruzi (TcGAL) expressed in E. coli were kindly provided by Prof. W.J.H. van Berkel (Wageningen University, Wageningen, The Netherlands). The recombinant expression of AtGALDH and TcGAL in E. coli was performed accordingly to [5,11].

Briefly, AtGALDH-His6 was expressed in E. coli BL21(DE3) cells as soluble cytoplasmic protein. The highest levels of expression were found after $16 \mathrm{~h}$ of induction with $0.4 \mathrm{~mm}$ isopropyl thio- $\beta$-d-galactoside (IPTG) at $37^{\circ} \mathrm{C}$. The recombinant protein was purified to apparent homogeneity by two successive chromatographic steps, by using Ni-NTA agarose and Q-Sepharose columns. Approximately $200 \mathrm{mg}$ of recombinant AtGALDH protein could be purified from a $12 \mathrm{~L}$ batch culture containing $58 \mathrm{~g}$ of cells (wet weight). The final preparation had a specific activity of $76 \mathrm{U} / \mathrm{mg}$.

The recombinant expression of TcGAL from T. cruzi was performed accordingly to [5], and the $\mathrm{pTrcHisC}$ vector containing the TcGAL gene (from T. cruzi X10/6 clone) was used. For enzyme production, E. coli BL21(DE3) cells harboring the pBAD-TcGAL plasmid were grown in Luria-Bertani (LB) medium supplemented with $100 \mu \mathrm{g} / \mathrm{mL}$ ampicillin until the OD600 reached $\sim 0.6$. The expression of TcGAL was induced by the addition of 
$0.02 \%$ L-arabinose, and the incubation was continued for $16 \mathrm{~h}$ at $28^{\circ} \mathrm{C}$. The cells were resuspended in $50 \mathrm{mM}$ Tris- $\mathrm{HCl}, 5 \mathrm{mM}$ DTT, $\mathrm{pH}$ 7.4, supplemented with 1 tablet of minicomplete protease inhibitor, $10 \mathrm{mM} \mathrm{MgCl}_{2}$ and DNase I and subsequently passed twice through a pre-cooled French Pressure cell at 10,000 psi. The resulting homogenate was centrifuged at $25,000 \times g$ for $30 \mathrm{~min}$ at $4{ }^{\circ} \mathrm{C}$ and the insoluble inclusion bodies were collected.

\subsection{Refolding of TcGAL Inclusion Bodies}

Refolding of TcGAL was performed according to [5] with slight modifications. The insoluble material collected after cell lysis was washed with $6 \%$ Triton X-100 containing $60 \mathrm{mM}$ EDTA and 1.5 M NaCl, pH 7.0 and dissolved in $6 \mathrm{M}$ guanidinium hydrochloride. Subsequently, the denaturant was removed by dialysis against $10 \mathrm{mM}$ sodium phosphate, $1 \mathrm{mM}$ DTT, $\mathrm{pH}=8.8$. A final dialysis step was performed against $10 \mathrm{mM}$ sodium phosphate, pH 8.8 to remove excess DTT. After dialysis, a turbid suspension of aggregated protein was obtained. This suspension was injected into the water-AOT-n-octane reverse micelles system containing $0.4 \mathrm{M}$ AOT and shaken intensively. The hydration degree, $\mathrm{W}_{0}=30$, was achieved by the addition $10 \mathrm{mM}$ sodium phosphate, $\mathrm{pH}$ 8.8. Oxidized and reduced glutathione solutions (100 mM GSSG and $300 \mathrm{mM} \mathrm{GSH}$ ) in $10 \mathrm{mM}$ sodium phosphate, $\mathrm{pH}$ 8.8 , were mixed and an aliquot of $10 \mu \mathrm{L}$ was added to $1 \mathrm{~mL}$ of $0.4 \mathrm{M}$ AOT solution in octane. Refolding of the enzyme was initiated by mixing $1.01 \mathrm{~mL}$ of micellar solution containing glutathiones with $200 \mu \mathrm{L}$ of TcGAL micellar solution and an aliquot $(10 \mu \mathrm{L})$ of FAD cofactor solution in water (10-fold molar excess to enzyme concentration). The resultant solution was shaken for $10 \mathrm{~min}$ yielding a transparent TcGAL-containing system. The final micellar protein concentration was $1-2 \mathrm{mg} / \mathrm{mL}$.

2.5. Optimization of the Micellar Approach for the Determination of AtGALDH and TcGAL Activity: Spectral Properties of Electron Acceptors of AtGALDH and Selection of the Conditions of the Assay

Spectra of the oxidized and reduced forms (in aqueous and micellar media) of following electron acceptors (EAs) of AtGALDH were obtained: phenazine methosulfate, cytochrome C, 1,4-benzoquinone, tetramethylphenylenediamine. Reduced EAs forms were obtained by the addition of a 5-fold excess of $\mathrm{NaBH}_{4}$. Aqueous and micellar media were a phosphate buffer with $\mathrm{pH} 8.8$ (pH optimum of AtGALDH [18]) and a solution of $0.1 \mathrm{M}$ AOT in n-octane (hydration degree $\mathrm{W}_{0}=23$ corresponding to the activity optima for AtGALDH [5]), respectively. Based on the obtained data two main parameters of each EA were determined: the wavelength corresponding to the maximum difference $\varepsilon_{(\mathrm{Ox}-\mathrm{Red})}$ in the molar absorption coefficients of the oxidized and reduced forms of the EA used, and the maximum difference of the absorption coefficients.

The catalytic parameters of systems using these EAs were calculated, including $\mathrm{K}_{\mathrm{M}}$, $\mathrm{V}_{\text {max }}$ and signal/background ratio (initial velocities were calculated using a molar difference absorption coefficient $\varepsilon_{(\mathrm{Ox}-\mathrm{Red})}$ for each EA). Based on spectral properties and the catalytic parameters, the optimal EA was chosen (both in the case of aqueous and micellar media) and the conditions of the spectrophotometric assay were selected. The selected conditions were: $\mathrm{pH}$ 8.8., $120 \mu \mathrm{M}$ phenazine methosulfate was used as an EA in combination with $120 \mu \mathrm{M}$ 2,6-dichlrophenolindophenol dye (wavelength $=550 \mathrm{~nm}$ in micellar media and $600 \mathrm{~nm}$ in aqueous media), the concentrations of AtGALDH and TcGAL were $6 \times 10^{-9} \mathrm{M}$ and $3.5 \times 10^{-5} \mathrm{M}$, respectively, the concentrations of L-galactono-1,4-lactone and D-arabinono-1,4-lactone were kept constant in the inhibition analysis $(1 \mathrm{mM})$ and varied during other experiments, as indicated in the corresponding sections. The improved approach was tested for the inhibition analysis of AtGALDH (in both aqueous and micellar media) and TcGAL (in micellar medium).

\subsection{The Effect of AOT Concentration on the AtGALDH Activity in the Reverse Micelles System}

To study the effect of the concentration of AOT on the AtGALDH activity in the reverse micelles system, solutions of reverse micelles of AOT were prepared in the range of concentration of AOT from 0.1 to $0.4 \mathrm{M}$. The hydration degree $\mathrm{W}_{0}=28$ corresponding 
to one of the optima of ATGALDH was kept constant by adding an appropriate volume of phosphate buffer ( $\mathrm{pH} 8.8$ ), which was added to the constant volume of solution of AOT in n-octane. All measurements were performed at $\mathrm{pH} 8.8$ using the combination of $120 \mu \mathrm{M}$ phenazine methosulfate as an EA and $120 \mu \mathrm{M}$ 2,6-dichlrophenolindophenol dye. The concentrations of AtGALDH and L-galactone-1,4-lactone were $6 \times 10^{-9} \mathrm{M}$ and $1 \mathrm{mM}$, respectively.

\subsection{Inhibition of AtGALDH and TcGAL by Lycorine}

Studies of the inhibition effect of AtGALDH and TcGAL by lycorine were carried out in aqueous and micellar media using the improved approach. Aqueous and micellar media were a phosphate buffer with $\mathrm{pH} 8.8$ and a solution of $0.1 \mathrm{M}$ AOT in n-octane (at different hydration degrees), respectively.

The dependence of inhibition of AtGALDH on concentration of lycorine in aqueous media was studied at varying concentrations of the inhibitor in the range of $0-800 \mu \mathrm{M}$. The mechanism of inhibition of AtGALDH in aqueous media was investigated with varying concentrations of L-galactone-1,4-lactone in the range of $0.12-1.01 \mathrm{mM}$ at fixed concentrations of lycorine $(0,0.5$ and $1 \mathrm{mM})$.

The dependence of the inhibition of AtGALDH on hydration degree in micellar media was studied with varying hydration degrees of $W_{0}$ in the range of 24-44, at a constant concentration of lycorine $(800 \mu \mathrm{M})$.

The dependence of the inhibition of TcGAL on the concentration of lycorine in micellar media was studied with varying concentrations of lycorine in the range of 0-800 $\mu \mathrm{M}$, at two hydration degrees, $W_{0}=22$ and $W_{0}=28$, corresponding to optima of TcGAL activity (which were assigned to monomeric and dimeric forms of TcGAL in reverse micelles [5]).

The concentrations of AtGALDH and TcGAL were $6 \times 10^{-9} \mathrm{M}$ and $3.5 \times 10^{-5} \mathrm{M}$, respectively, in all experiments. All measurements were performed at $\mathrm{pH} 8.8$ using the combination of $120 \mu \mathrm{M}$ phenazine methosulfate as an EA and $120 \mu \mathrm{M}$ 2,6-dichlrophenolindophenol dye. The concentrations of L-galactone-1,4-lactone and D-arabinono-1,4-lactone were $1 \mathrm{mM}$ in all measurements except for the study of the mechanism of inhibition of AtGALDH by lycorine.

\section{Results and Discussion}

\subsection{Spectral Properties of Electron Acceptors of AtGALDH}

Our aim was the improvement of the micellar approach to the inhibition analysis of membrane enzymes with AtGALDH and TcGAL as examples. Spectrophotometric determination was rather simple, but not effective enough, and needed to be improved using previously unstudied EAs. In general, the spectrophotometric determination of AtGALDH and TcGAL activity followed the reduction of EA at a wavelength corresponding to the maximum difference in the molar absorption coefficients of the oxidized and reduced forms of the EA used. The optimal wavelength was determined from the spectra of the oxidized and reduced forms of the EA in a specific medium (aqueous, micellar, etc.).

In contrast to the case of aqueous medium, the determination of AtGALDH activity in the reverse micelles system required the use of artificial EAs because of the strong interaction of cytochrome $\mathrm{C}$ with the AOT interface [12], which leads to loss of the cytochrome C secondary structure [13]. The authors of [5] used 1,4-benzoquinone as an EA for AtGALDH in a micellar medium, which is still the only reported spectrophotometric method for the determination of the AtGALDH activity in reverse micelles systems. However, the use of 1,4-benzoquinone is complicated by the need to take into account the rate of the background reaction (low signal-to-noise ratio) [5]. In addition, it should be noted that it is impossible to use 1,4-benzoquinone in strongly alkaline media (where the $\mathrm{pH}$ optimum of AtGALDH is usually observed), since significant autooxidation of this EA occurs at $\mathrm{pH}>7.2$ [19]. Thus, it is necessary to study other artificial EAs for the development of methods to determine AtGALDH activity. 
In this work, the spectra of two EAs for AtGALDH (Figure 2) in an AOT reverse micelles system were obtained and characterized for the first time: phenazine methosulfate (previously used only for aqueous media [11]) and tetramethylphenylenediamine (which has a similar structure to 1,4-benzoquinone and was used to determine peroxidase activity [20]). Reduced EA forms were obtained by the addition of a 5-fold excess of $\mathrm{NaBH}_{4}$. Measurements with the above EAs in water, as well as with cytochrome $\mathrm{C}$, were used as controls. The Figure $2 \mathrm{c}$ shows the spectra of tetramethylphenylenediamine in both media_aqueous and micellar (AOT) - to illustrate the influence of the medium on the spectral properties of the EAs.
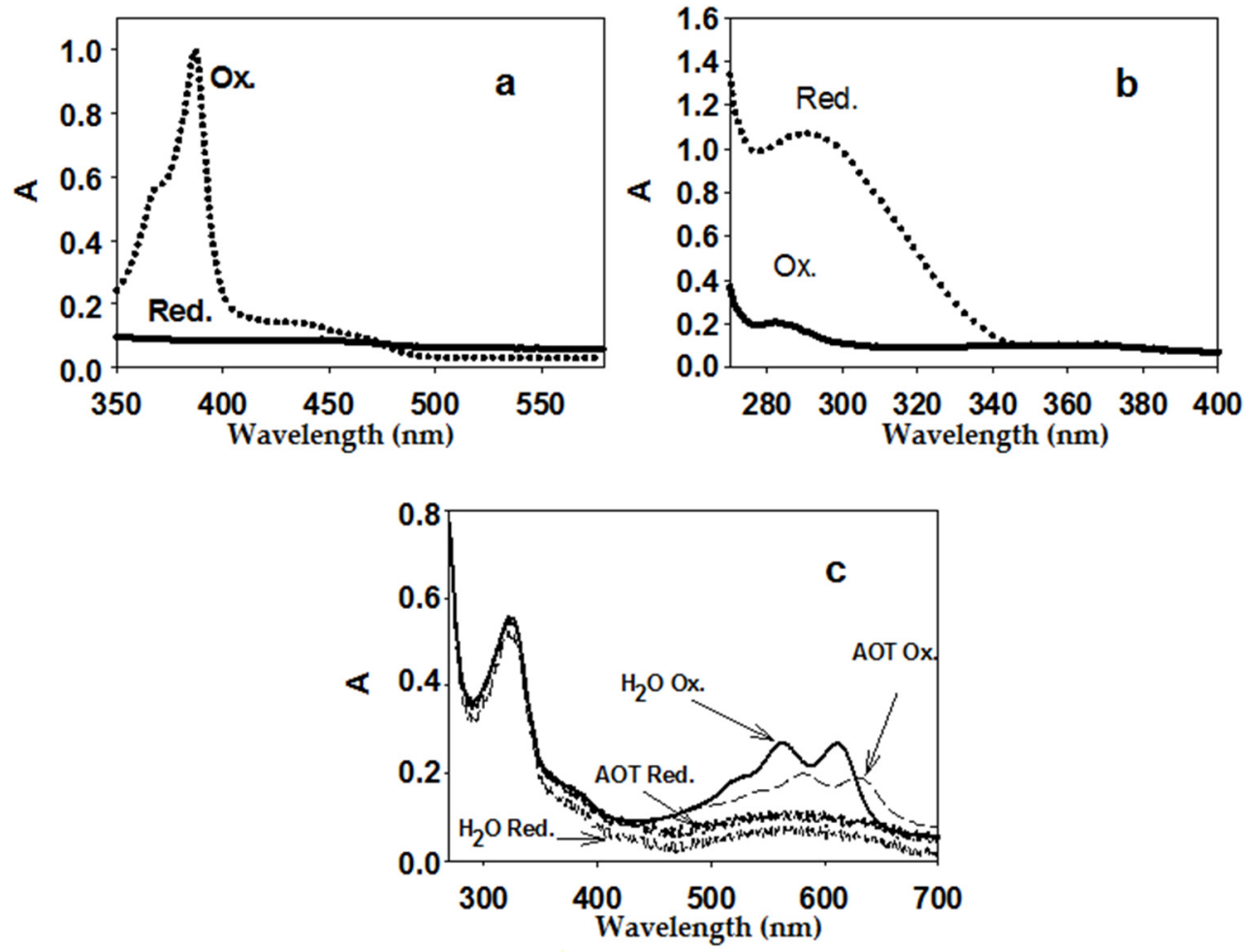

Figure 2. Spectra of oxidized and reduced EA forms: $50 \mu \mathrm{M}$ phenazine methosulfate (a) (micelles), $200 \mu \mathrm{M}$ 1,4-benzoquinone; (b) (micelles), $25 \mu \mathrm{M}$ tetramethylphenylenediamine; (c) aqueous medium and micelles. Aqueous medium was $25 \mathrm{mM}$ phosphate buffer $(\mathrm{pH} 8.8)$, micelles were $0.1 \mathrm{M}$ AOT in n-octane (with the addition of the required volume of phosphate buffer to obtain the hydration degree $\mathrm{W}_{0}=23$, at which one of the activity optima for AtGALDH is observed [5]).

Based on the obtained spectra of the oxidized and reduced forms of EAs, we chose the wavelengths at which the largest difference in absorption (and, accordingly, the maximum difference in the molar absorption coefficients) of the oxidized and reduced forms of EAs in reverse micelles was observed. Then, at these wavelengths, the absorption of the oxidized and reduced forms of the EAs were measured at various concentrations of EAs, and the difference in the molar absorption coefficients of the oxidized and reduced forms for each EA ( $\left.\varepsilon_{(\mathrm{Ox}-\mathrm{Red})}\right)$ were determined. The optimal wavelengths for aqueous (control) and micellar media and the calculated differences in molar absorption coefficients are shown in Table 1.

Cytochrome $C$ and 1,4-1,4-benzoquinone (both in aqueous medium) were used as controls in the experiments with phenazine methosulfate and tetramethylphenylenediamine. Calculated differences $\left(\varepsilon_{(\mathrm{Ox}-\mathrm{Red})}\right)$ in the molar absorption coefficients of the oxidized and reduced forms of these EAs were in good correlation with those given in work [5]. It should be noted that a combination of an EA and a special indicator can also be used. For example, the use of the combination of phenazine methosulfate and 2,6-dichlorophenolindophenol (DCPIP) dye: not being an EA for AtGALDH, DCPIP changes its own color, reacting with 
the ascorbate formed during the reaction, so the process can be followed spectrophotometrically at a wavelength of $600 \mathrm{~nm}$ (it is still necessary to use EA besides DCPIP, since EA is the second substrate, the role of which, in this case, is played by phenazine methosulfate).

Table 1. Parameters ** of electron acceptors for AtGALDH and 2,6-dichloro-phenolindophenol dye in aqueous (control) and micellar media (aqueous medium was phosphate buffer, $\mathrm{pH} 8.8$, micellar was $0.1 \mathrm{M}$ AOT in n-octane, hydration degree $\mathrm{W}_{0}=23$ ).

\begin{tabular}{|c|c|c|c|c|c|}
\hline EA & Media & $\begin{array}{l}\text { Wavelength } \\
\text { (nm) }\end{array}$ & $\begin{array}{l}\mathcal{E}_{(\text {Ox-Red })}{ }^{* * *} \\
\mathbf{M}^{-1} \mathbf{c m}^{-1}\end{array}$ & $\mathcal{E}_{(\mathrm{Ox})}, \mathrm{M}^{-1} \mathrm{~cm}^{-1}$ & $\begin{array}{c}\left.\mathcal{E}_{(\mathrm{Red})}\right) \\
\mathbf{M}^{-1} \mathrm{~cm}^{-1}\end{array}$ \\
\hline Cytochrome $C^{*}$ & Water * & 550 & 21,000 & 7200 & 28,200 \\
\hline \multirow{2}{*}{ 1,4-benzoquinone } & Micelles & 290 & 2300 & 750 & 3050 \\
\hline & Water & 290 & 2300 & 800 & 3100 \\
\hline \multirow{2}{*}{ Phenazine methosulfate } & Micelles & 388 & 25,000 & 27,100 & 2100 \\
\hline & Water & 388 & 25,000 & 26,800 & 1800 \\
\hline \multirow{2}{*}{ 2,6-dichlorophenolindophenol } & Micelles & 550 & 11,900 & 14,300 & 2400 \\
\hline & Water & 600 & 20,600 & 21,700 & 1100 \\
\hline \multirow{2}{*}{ Tetramethylphenylene-diamine } & Micelles & 605 & 7800 & 12,200 & 4400 \\
\hline & Water & 590 & 8400 & 11,500 & 3100 \\
\hline
\end{tabular}

* Does not function in reverse micelles system. ${ }^{* *}$ The parameters have maximal error values of $10 \% .{ }^{* * *}$ Absolute values of the difference in the molar absorption coefficients of the oxidized and reduced forms of EAs.

In this work the differences in molar absorption coefficients of the oxidized and reduced forms of phenazine methosulfate and tetramethylphenylenediamine in the AOT reverse micelles system were calculated for the first time. The difference in the molar absorption coefficients in the case of the phenazine methosulfate was the same in both media $\left(25,000 \mathrm{M}^{-1} \mathrm{~cm}^{-1}\right.$ in aqueous and micellar media), although it differed slightly in the case of tetramethylphenylenediamine $\left(8400\right.$ and $7800 \mathrm{M}^{-1} \mathrm{~cm}^{-1}$ in aqueous and micellar media, respectively).

For the reasons mentioned above (interaction with the AOT interface), the use of cytochrome $\mathrm{C}$ as an EA for AtGALDH in a micellar medium is impossible. Among the rest of EAs presented in Table 1, the largest value of the maximum difference in molar absorption coefficients was observed for phenazine methosulfate $\left(25,000 \mathrm{M}^{-1} \mathrm{~cm}^{-1}\right.$ at $388 \mathrm{~nm}$ ), which should provide the best determination of the AtGALDH activity (higher sensitivity). However, the efficiency of the EA in terms of the determination of the enzyme activity also strongly depended on the ratio of the rates of the main and background (in the absence of the enzyme) reactions, which became the subject of further study.

\subsection{Development of the Inhibition-Analysis-Approach-Based Micellar Method}

To estimate the applicability of the studied EAs, the main catalytic parameters of the systems were determined, including the ratio of the rates of the main and background reactions, the maximum initial rate, Michaelis constants $\mathrm{K}_{\mathrm{M}}$ (for the main and the second substrate). The rate of the main reaction for each EA was calculated as the difference between the rates in the presence and in the absence of AtGALDH (background response). To determine $\mathrm{K}_{\mathrm{M}}$ for the substrate and EA (second substrate), as well as the maximum rate, a series of experiments were carried out by varying the concentration of each of the substrates in turn, and the corresponding Lineweaver-Burk plots were analyzed. For each $\mathrm{EA}$, the $\mathrm{pH}$ of both media (aqueous and micellar) was 8.8 (corresponding to the $\mathrm{pH}$ optimum of AtGALDH [18]), with the exception of 1,4-benzoquinone, for which a $\mathrm{pH}$ of 7.8 was chosen (a more alkaline medium causes auto-oxidation of this EA [19], and leads to a decrease in the signal-to-noise ratio). Examples of the plots used for calculations of the catalytic parameters are shown in Figure 3. 

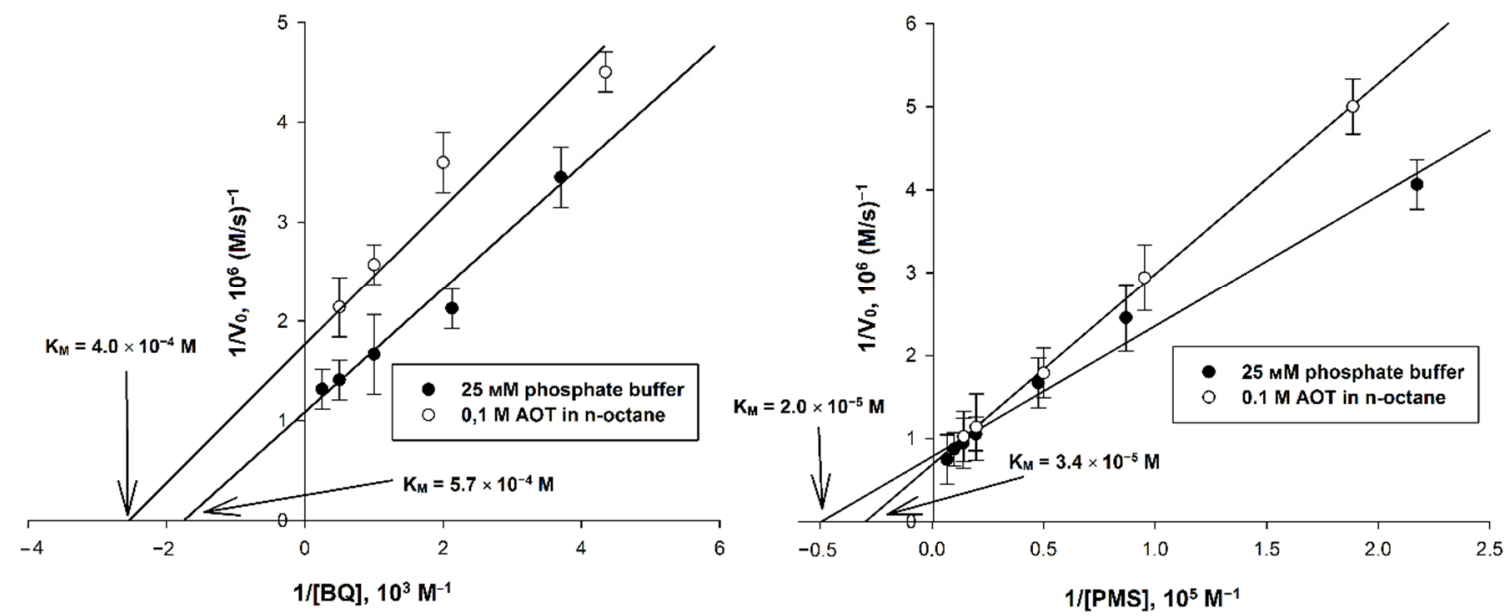

Figure 3. Double reciprocal plots for systems using 1,4-benzoquinone and phenazine methosulfate (with varying EA concentration), for which media with $\mathrm{pH} 7.8$ and 8.8 were used, respectively. The concentrations of AtGALDH and and L-galactone-1,4-lactone were $6 \times 10^{-9} \mathrm{M}$ and $1 \mathrm{mM}$, respectively. Values are presented as the mean $\pm \mathrm{SD}$ of three experiments.

The calculated catalytic parameters, as well as the optimal wavelengths and the corresponding differences in the molar absorption coefficients of the oxidized and reduced forms of EAs, are presented in Table 2.

Table 2. Parameters of the studied EAs in aqueous and micellar media. The enzyme concentration was $6 \times 10^{-9} \mathrm{M}$. In experiments to determine the $\mathrm{K}_{\mathrm{M}}$ for EA, the concentration of L-galactone-1,4-lactone $(1 \mathrm{mM})$ was kept constant, while varying the EA concentration as follows: $2-150 \mu \mathrm{M}$ for cytochrome C, $0.1-4 \mathrm{mM}$ for 1,4-benzoquinone (water and micelles), $0.1-8 \mathrm{mM}$ for tetramethylphenylenediamine (water and micelles), 2-150 and 1-70 $\mu \mathrm{M}$ for phenazine methosulfate (water and micelles, respectively). In experiments to determine the $\mathrm{K}_{\mathrm{M}}$ for the main substrate, the concentration of L-galactone-1,4-lactone was varied in the range of 0.1-2 mM, while maintaining constant EA concentrations: $100 \mu \mathrm{M}$ for cytochrome $\mathrm{C}$ (water), $2 \mathrm{mM}$ for 1,4-benzoquinone (water and micelles), $25 \mu \mathrm{M}$ for tetramethylphenylenediamine, $50 \mu \mathrm{M}$ for phenazine methosulfate (water and micelles), as well as $70 \mu \mathrm{M}$ for phenazine methosulfate and $100 \mu \mathrm{M}$ for dichlorophenolindophenol when used together (water and micelles). Values are the mean \pm SD of three experiments.

\begin{tabular}{|c|c|c|c|c|c|c|}
\hline Electron Acceptor & Medium & $\mathrm{K}_{\mathrm{M}(\mathrm{EA}), \mathrm{M}}$ & $\mathrm{K}_{\mathrm{M} \text { (substrate), }} \mathrm{M}$ & $\mathrm{V}_{(\max )}, \mathrm{M} / \mathrm{s}$ & $\begin{array}{c}\mathbf{V}_{(\max )} / \\
\mathbf{V}_{\text {(background) }}\end{array}$ & $\begin{array}{c}\mathcal{E}_{(\mathrm{Ox}-\mathrm{Red})} \\
\mathrm{M}^{-1} \mathrm{~cm}^{-1} \\
(\lambda, \mathrm{nm})\end{array}$ \\
\hline Cytochrome C & Water & $(7.1 \pm 0.2) \times 10^{-5}$ & $(1.7 \pm 0.1) \times 10^{-4}$ & $(1.3 \pm 0.6) \times 10^{-6}$ & 460 & $21,000(550)$ \\
\hline \multirow{2}{*}{ 1,4-benzoquinone } & Water & $(5.7 \pm 0.4) \times 10^{-4}$ & $(1.9 \pm 0.3) \times 10^{-4}$ & $(9.5 \pm 0.4) \times 10^{-7}$ & 7 & $2300(290)$ \\
\hline & Micelles & $(4.0 \pm 0.3) \times 10^{-4}$ & $(2.0 \pm 0.3) \times 10^{-4}$ & $(5.0 \pm 0.7) \times 10^{-7}$ & 6 & $2300(290)$ \\
\hline \multirow{2}{*}{ Tetramethylphenylenediamine } & Water & $(1.0 \pm 0.3) \times 10^{-3}$ & $(2.0 \pm 0.2) \times 10^{-4}$ & $(5.0 \pm 0.3) \times 10^{-7}$ & 2.7 & $8400(590)$ \\
\hline & Micelles & $(2.7 \pm 0.3) \times 10^{-3}$ & $(2.7 \pm 0.6) \times 10^{-4}$ & $(2.6 \pm 0.7) \times 10^{-6}$ & 13 & $7800(605)$ \\
\hline \multirow{2}{*}{ Phenazine methosulfate } & Water & $(2.0 \pm 0.3) \times 10^{-5}$ & $(2.1 \pm 0.2) \times 10^{-4}$ & $(1.3 \pm 0.1) \times 10^{-6}$ & 6 & $25,000(388)$ \\
\hline & Micelles & $(3.4 \pm 0.2) \times 10^{-5}$ & $(2.3 \pm 0.2) \times 10^{-4}$ & $(1.5 \pm 0.1) \times 10^{-6}$ & 10.7 & $25,000(388)$ \\
\hline $\begin{array}{l}\text { Phenazine methosulfate } \\
+2,6 \text {-dichlorophenolindophenol }\end{array}$ & Micelles & $(2.8 \pm 0.1) \times 10^{-5}$ & $(2.1 \pm 0.2) \times 10^{-4}$ & $(1.2 \pm 0.1) \times 10^{-6}$ & 160 & $11,900(550)$ \\
\hline
\end{tabular}

As mentioned in a previous paragraph, 1,4-benzoquinone has been already applied as an EA for AtGALDH [5], but the use of this EA has significant disadvantages (low signal-to-noise ratio and auto-oxidation of the EA in strong alkaline media). The use of other EAs studied in this work provides some benefits: having approximately the same signal-to-noise ratio (with the exception of a combination of phenazine methosulfate and 2,6dichlorophenolindophenol), these EAs give the opportunity to perform measurements at 
the $\mathrm{pH}$ optimum of AtGALDH (pH 8.8). Besides this, comparison of 1,4-benzoquinone and other studied EAs has demonstrated that 1,4-benzoquinone has the smallest difference in the molar absorption coefficients of the oxidized and reduced forms. The largest difference in the molar absorption coefficients in both aqueous and micellar media was observed in case of phenazine methosulfate $\left(25,000 \mathrm{M}^{-1} \mathrm{~cm}^{-1}\right.$ at $\left.388 \mathrm{~nm}\right)$. However, the data obtained indicate that phenazine methosulfate, despite having the largest value of the maximum difference in molar absorption coefficients, is less suitable for the determination of the AtGALDH activity in reverse micelles, compared to the combination of phenazine methosulfate and dichlorophenolindophenol, because of a much lower ratio of rates of the main and background reactions (10.7 versus 160, while the differences in the molar absorption coefficients differed by only 2 times).

To compare other parameters of EAs, and accordingly to reveal which EAs are the most suitable for the determination of AtGALDH activity in reverse micelles systems, it should be noted that the medium (aqueous or micellar) has no significant impact on $\mathrm{K}_{\mathrm{M}}$, both in the case of EAs and in the case of the main substrate (the observed insignificant differences in $\mathrm{K}_{\mathrm{M}}$ may be due to redistribution of the substrate between water and organic phases). However, in the micellar medium, a slight increase in the ratio of the main and background reactions is possible (for phenazine methosulfate and tetramethylphenylenediamine). Both $\mathrm{K}_{\mathrm{M}}$ for the main substrate and $\mathrm{V}_{\max }$ differed insignificantly for the studied EAs (mean values were $2 \times 10^{-4} \mathrm{M}$ and $1 \times 10^{-6} \mathrm{M} / \mathrm{s}$, respectively), while $\mathrm{K}_{\mathrm{M}}$ for EAs was in the range of $10^{-3}-10^{-5} \mathrm{M}$ (the smallest and the largest value are $2.0 \times 10^{-5} \mathrm{M}$ and 2.7 $\times 10^{-3} \mathrm{M}$ for phenazine methosulfate and tetramethylphenylenediamine, respectively). Moreover, the most important parameters in terms of determination of the enzyme activity are signal-to-noise ratio and difference in the molar absorption coefficients of the oxidized and reduced forms of EA used.

Thus, with comparable values of $K_{M}$ (for main substrate and EA) and $V_{\max }$, preference should be given to EA, which combines the largest maximum difference in the molar absorption coefficients of the oxidized and reduced forms and the maximum ratio of the rates of the main and background reactions. Among the studied EAs, this requirement was satisfied by the combination of phenazine methosulfate and the 2,6-dichlorophenolindophenol dye (all other EAs, except for phenazine methosulfate separately, had a smaller maximum difference in absorption coefficients, and phenazine methosulfate itself had a small ratio of the rates of the main and background reactions). Besides this, $K_{M}$ for this combination (as well as to phenazine methosulfate separately), was also one of the lowest among the studied EAs.

Thus, based on the obtained data, the following conditions of the assay were selected: $\mathrm{pH} 8.8,120 \mu \mathrm{M}$ phenazine methosulfate was used as an EA in combination with $120 \mu \mathrm{M}$ 2,6-dichlrophenolindophenol dye (wavelength $=550 \mathrm{~nm}$ in micellar media and $600 \mathrm{~nm}$ in aqueous media), the concentration of AtGALDH was $6 \times 10^{-9} \mathrm{M}$, the concentration of L-galactono-1,4-lactone was kept constant in inhibition analysis $(1 \mathrm{mM})$ and varied during other experiments, as indicated in the corresponding sections. Suggesting the similarity of TcGAL and AtGALDH, this approach was also applied to the inhibition analysis of $3.5 \times 10^{-5} \mathrm{M}$ TcGAL by lycorine using $1 \mathrm{mM}$ D-arabinono-1,4-lactone instead of L-galactono-1,4-lactone.

The improved approach described above has several advantages in comparison with the method given in [5], such as the possibility to perform experiments at the $\mathrm{pH}$ optimum of the enzyme ( $\mathrm{pH} 8.8$ ), and much higher signal/background ratio (so one need not take into consideration the rate of the background reactions). The method can be applied for the determination of AtGALDH and TcGAL activities, as well as for inhibition analysis of these enzymes.

\subsection{Effect of AOT Concentration on AtGALDH Activity in the Reverse MICELLES System}

It has been found that an enzyme in a reverse micelle system normally shows a bell-shaped dependence of its catalytic activity on the degree of surfactant hydration 
$\left.\left(\mathrm{W}_{0}=\mathrm{H}_{2} \mathrm{O}\right] /[\mathrm{AOT}]\right)$ with an optimum at conditions under which the enzyme size corresponds to the size of the micelle inner cavity [15]. Optima in the activity of AtGALDH was observed at $\mathrm{W}_{0}=23$ and 28 , which is associated with its functioning in monomeric and dimeric forms, respectively [5], which was shown by the sedimentation method (A.A. Chudin, E.V. Kudryashova, unpublished results).

Another important parameter that can also influence the AtGALDH activity is the concentration of the micelle-forming agent at a fixed hydration degree. In the general case, an increase in the surfactant concentration (in our case, AOT) leads to an increase in the number of micelles in the system [21], which, on the one hand, can contribute to better solubization of the protein and substrates (increased enzyme activity), and on the other hand, lead to a more pronounced interaction of the enzyme with the interface, which can result in a decrease in the activity [22]. At the same time, the activity of some enzymes, mainly in the aqueous phase, does not depend on the surfactant concentration; therefore, the question of the effect of the AOT concentration on the AtGALDH activity in the reverse micelles system is very important. This should reveal whether the enzyme interacts with the interface in micelles, or if it is mainly located in the aqueous phase.

We investigated the effect of AOT concentration in the range of $0.1-0.4 \mathrm{M}$ on the AtGALDH activity in the reverse micelles system, maintaining the hydration degree $\mathrm{W}_{0}=28$, at which the enzyme functions in a dimeric form. The system composition was: $120 \mu \mathrm{M}$ phenazine methosulfate in combination with $120 \mu \mathrm{M}$ 2,6-dichlorophenolindophenol, AtGALDH $\left(6 \times 10^{-9} \mathrm{M}\right)$ and L-galactone-1,4-lactone $(1 \mathrm{mM})$.

It was found that the AtGALDH activity insignificantly depends on the concentration of AOT (the AtGALDH activity is in the range of $(8-10.5) \times 10^{-7} \mathrm{M} / \mathrm{s}$ and the average value is $9.2 \times 10^{-7} \mathrm{M} / \mathrm{s}$ ); that is, it can be concluded that AtGALDH is predominantly in the aqueous phase and partly interacts with this surfactant (AOT) [23].

\subsection{Sequence Analysis of AtGALDH, TcGAL and Related Enzymes}

Lycorine is used as a drug and has long been known as a highly specific inhibitor of L-galactone-1,4-lactone dehydrogenases from various sources such as Ipomoea batatas [9] and Zea mays [10], so it is reasonable to compare the amino acid sequences of these dehydrogenases, AtGALDH and TcGAL, studied in this work.

By performing the multiple alignment of TcGAL, AtGALDH, and two L-galactone-1,4lactone dehydrogenases from Ipomoea batatas and Zea mays, correspondently (for the last two the inhibitory effect of lycorine has been described in the literature $[9,10])$, we found the presence of common Glu and Arg residues in the active centers of the enzymes (circled in red in Figure 4) and a high similarity of the environments of these amino acid residues (similar positions are highlighted in dark and light gray, respectively). In addition, there was a conserved Cys residue (circled in red in Figure 4), which, as shown by the authors [6], also plays an important role in the catalysis of L-galactone-1,4-lactone dehydrogenase.

AtGALDH 400 IbGALDH 374 ZmGALDH 373 TcGAL $\quad 305$
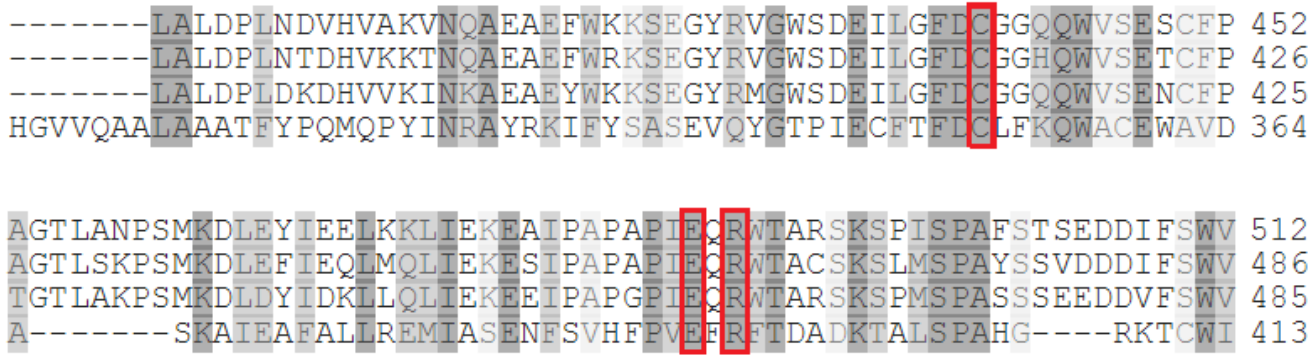

Figure 4. Clustal Omega multiple sequence alignment of AtGALDH, TcGAL and two L-galactone-1,4lactone dehydrogenases from Ipomoea batatas (IbGALDH) and Zea mays (ZmGALDH). The accession numbers used are as follows: AtGALDH, Q9SU56; IbGALDH, Q9ZWJ1; ZmGALDH, A0A3L6FX76; TcGAL, A0A2V2VEI5. 
To identify the enzymes most similar to each other (studied in this work and described in the literature), we also performed pairwise alignments (using the same accession numbers given in the description for Figure 4). Thus, AtGALDH shows 53.8 and $73 \%$ sequence identity to ZmGALDH and IbGALDH, respectively. In other words, AtGALDH and two L-galactone-1,4-lactone dehydrogenases described in the literature (ZmGALDH and IbGALDH) not only had common residues in the active center, but had a high degree of homology in general, which suggests a similar effect of lycorine on the AtGALDH activity. At the same time, TcGAL showed just 13.6 and $16.9 \%$ sequence identity to ZmGALDH and IbGALDH. However, a high degree of homology near the active centers of these enzymes (Figure 4) allows us to suggest there is an inhibitory effect of lycorine on TcGAL activity too.

Thus, based on the above similarities, one can expect a similar inhibitory effect of lycorine on AtGALDH and TcGAL activities.

\subsection{Effect of Inhibition of AtGALDH Activity by Lycorine in Aqueous Medium}

The study of the effect of lycorine on AtGALDH activity was carried out in $25 \mathrm{mM}$ phosphate buffer with a $\mathrm{pH}$ of 8.8, corresponding to the $\mathrm{pH}$ optimum of the enzyme [18]. Since typical concentrations of lycorine used in inhibition studies are in the range from several tens to hundreds of $\mu \mathrm{M}[9,10]$, in this work, inhibitor concentrations from 0 to $680 \mu \mathrm{M}$ were used for an aqueous medium (Figure 5).

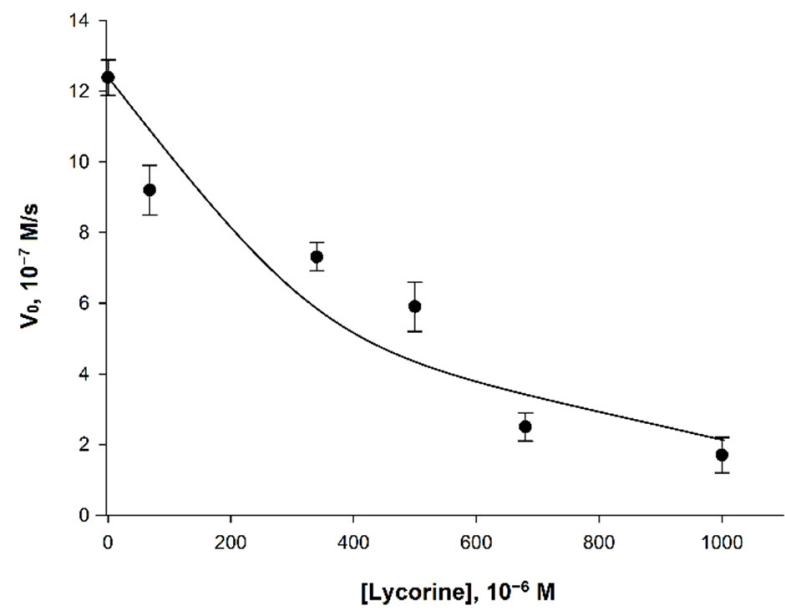

Figure 5. Dependence of the initial velocity $\left(\mathrm{V}_{0}\right)$ on the concentration of lycorine. Measurements were performed in $25 \mathrm{mM}$ phosphate buffer at $\mathrm{pH} 8.8$ using a combination of $120 \mu \mathrm{M}$ phenazine methosulfate and $120 \mu \mathrm{M}$ dichlorophenolindophenol. The concentrations of AtGALDH and L-galactone1,4-lactone were $6 \times 10^{-9} \mathrm{M}$ and $1 \mathrm{mM}$, respectively. Values are presented as the mean $\pm \mathrm{SD}$ of three experiments.

The observed effect of inhibition of AtGALDH activity was less pronounced than for L-galactone-1,4-lactone dehydrogenases from other sources (Table 3). For example, L-galactone-1,4-lactone dehydrogenase from Ipomoea batatas already had a complete loss its activity at the lycorine concentration of $340 \mu \mathrm{M}$ [9], while the inhibitory effect of lycorine on AtGALDH activity was only $45 \%$ (at the same concentration of inhibitor).

Nevertheless, given the high specificity of the inhibitor [10] and its relatively low toxicity - as well as the fact that lycorine is an approved drug [24] - the inhibitory effect of lycorine seems to be significant enough to further study its effect on AtGALDH activity in membrane models (for example, AOT reverse micelles systems).

\subsection{Mechanism of Inhibition of AtGALDH Activity by Lycorine}

Although lycorine is known as an inhibitor of L-galactone-1,4-lactone dehydrogenases from various sources, there is no literature data that describes its mechanism of inhibition for these enzymes (including L-galactone-1,4-lactone dehydrogenases from Arabidopsis 
thaliana and Trypanosoma cruzi). Authors [25] suppose that the mechanism of inhibition of galactone- $\boldsymbol{\gamma}$-lactone oxidase by lycorine is non-competitive, since it quickly forms a stable bond with the enzyme (suppression of ascorbate synthesis from galactone- $\gamma$-lactone in vivo). However, these data refer to oxidases, and cannot be generalized for L-galactone1,4-lactone dehydrogenases, including AtGALDH. Thus, the mechanism of inhibition of L-galactone-1,4-lactone dehydrogenases (including that from Arabidopsis thaliana) by lycorine has not been previously described, and is studied in this work for the first time.

Table 3. Comparison of the inhibitory effect of lycorine on the activity of L-galactone-1,4-lactone dehydrogenases from various sources at approximately 50 and $90 \%$ inhibition.

\begin{tabular}{cccc}
\hline Source & Inhibition, $\%$ & Concentration of Lycorine, $\boldsymbol{\mu M}$ & Reference \\
\hline Zea mays & 50 & 2 & {$[10]$} \\
\hline Ipomoea batatas & 43 & 68 & {$[9]$} \\
\hline Arabidopsis thaliana & 52 & 500 & This work \\
\hline Zea mays & 91 & 10 & {$[10]$} \\
\hline Ipomoea batatas & 86 & 170 & {$[9]$} \\
\hline Arabidopsis thaliana & 86 & 800 & This work \\
\hline
\end{tabular}

In order to determine the mechanism of inhibition of AtGALDH by lycorine, the activity (initial rate) of AtGALDH was measured at concentrations of L-galactone-1,4lactone in the range of $0.12-1.01 \mathrm{mM}$ at fixed concentrations of the inhibitor $(0,0.5$ and $1 \mathrm{mM})$ in aqueous medium ( $25 \mathrm{mM}$ phosphate buffer, $\mathrm{pH} 8.8$ ). The corresponding LineweaverBurk plots are presented in Figure 6.

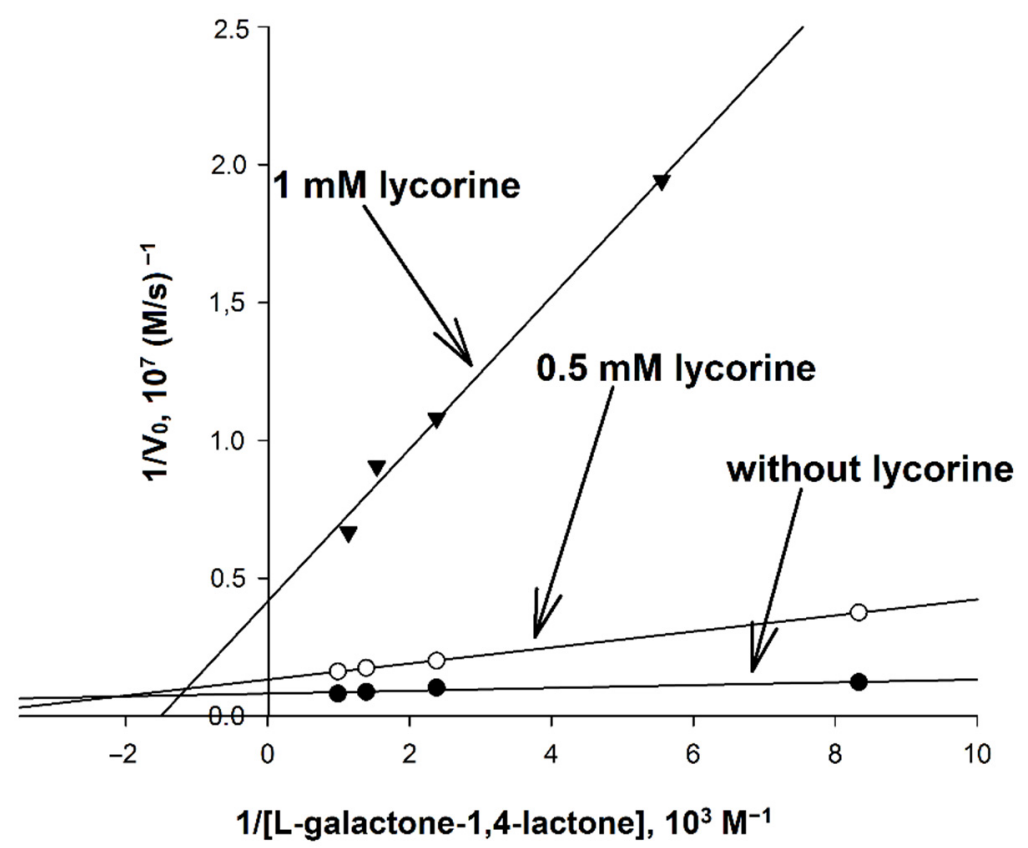

Figure 6. Determination of the mechanism of inhibition of AtGALDH by lycorine. The concentration of AtGALDH was $6 \times 10^{-9} \mathrm{M}$. Concentrations of L-galactone-1,4-lactone were in the range of $0.12-1.01 \mathrm{mM}$ at fixed concentrations of the inhibitor $(0,0.5$ and $1 \mathrm{mM})$. Measurements were performed in in aqueous medium $(25 \mathrm{mM}$ phosphate buffer, $\mathrm{pH}$ 8.8). The parameters have maximal error values of $10 \%$.

It was found that inhibition by lycorine affects both $\mathrm{K}_{\mathrm{M}}$ and $\mathrm{V}_{\max }$, and to an unequal extent, which indicates a mixed mechanism of inhibition. Based on the data obtained, 
the catalytic parameters of the systems were calculated (Table 4), including $\mathrm{K}_{\mathrm{I}}$ and $\mathrm{K}_{\mathrm{I}}{ }^{\prime}$ corresponding to the inhibition constants describing competitive and non-competitive components of mixed inhibition, respectively, as well as the so-called reduced inhibition

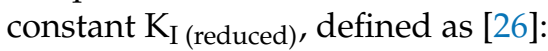

$$
\mathrm{K}_{\mathrm{I}(\text { reduced })}=\frac{\mathrm{K}_{\mathrm{I}} \mathrm{K}_{\mathrm{I}}^{\prime}}{\mathrm{K}_{\mathrm{I}}+\mathrm{K}_{\mathrm{I}}^{\prime}}
$$

Table 4. Catalytic parameters of the systems used to determine the mechanism of inhibition. Concentration of L-galactone-1,4-lactone was varied in the range of $0.12-1.01 \mathrm{mM}$ at fixed inhibitor concentrations $(0,0.5$, and $1 \mathrm{mM})$. The concentration of AtGALDH was $6 \times 10^{-9} \mathrm{M}$;. Values are presented as the mean \pm SD of three experiments.

\begin{tabular}{|c|c|c|c|c|c|c|}
\hline & $\mathrm{K}_{\mathrm{M}}(\mathrm{GL}), \mathrm{mM}$ & $\mathrm{k}_{\mathrm{cat}}, \mathrm{s}^{-1}$ & $\begin{array}{c}\mathrm{k}_{\mathrm{cat}} / \mathrm{K}_{\mathrm{M}} \\
\left(\mathrm{mM}^{-1} \cdot \mathrm{s}^{-1}\right)\end{array}$ & $\mathrm{K}_{\mathrm{I}}^{\prime}, \mathbf{m} \mathbf{M}$ & $\mathrm{K}_{\mathrm{I}}, \mathrm{mM}$ & $\mathrm{K}_{\mathrm{I} \text { (reduced), }} \mathrm{mM}$ \\
\hline In absence of lycorine & $0.063 \pm 0.02$ & $205 \pm 6$ & $3.3 \times 10^{3}$ & \multirow{3}{*}{$0.237 \pm 0.09$} & \multirow{3}{*}{$0.017 \pm 0.01$} & \multirow{3}{*}{$0.016 \pm 0.02$} \\
\hline $0.5 \mu \mathrm{M}$ lycorine & $0.220 \pm 0.08$ & $125 \pm 4$ & $5.7 \times 10^{2}$ & & & \\
\hline $1 \mu ; M$ lycorine & $0.665 \pm 0.05$ & $40 \pm 1$ & 6.0 & & & \\
\hline
\end{tabular}

Taking into account the ratio between $\mathrm{K}_{\mathrm{I}}$ and $\mathrm{K}_{\mathrm{I}}^{\prime}$, it can be concluded that the competitive component was predominant in comparison with the non-competitive one. In other words, lycorine acted as a mixed competitive inhibitor (which was also proved by the increasing of $\mathrm{K}_{\mathrm{M}}$ and decreasing $\mathrm{V}_{\max }$ ) according to the classification given in [27]. There is no literature data related to the constants of inhibition of L-galactone-1,4-lactone dehydrogenases (including those for AtGALDH) by lycorine, so the constants were determined for the first time, and this can shed a light on some aspects of the inhibition of L-galactone-1,4-lactone dehydrogenases by this inhibitor.

Considering the similarity of AtGALDH and the number of other enzymes (see Section 3.3), one can assume a similar mechanism of inhibition of L-galactone-1,4-lactone dehydrogenases from other sources (as well as TcGAL) by lycorine.

\subsection{Inhibition of AtGALDH and TcGAL Activity by Lycorine in a Micellar Medium}

In light of the fact that TcGAL is a membrane enzyme, it is reasonable to study the inhibition of the model AtGALDH using a membrane model, the AOT reverse micelle system. Such a system was previously used for TcGAL by the authors [5] for the refolding of TcGAL from inclusion bodies.

To study the inhibition of AtGALDH, 0.1 M AOT in n-octane was used, conducting experiments at the hydration degree $W_{0}$ in the range of 24-44 by adding the required volume of $25 \mathrm{mM}$ phosphate buffer ( $\mathrm{pH} 8.8$ ) and using a constant concentration of lycorine $(800 \mu \mathrm{M})$. The results are shown in Figure 7 . For comparison, Figure 7 also presents the results of measurements in the absence of lycorine at the different hydration degree $\mathrm{W}_{0}$, (the AtGALDH activity in the presence of lycorine is not completely suppressed at any $W_{0}$ ).

The dependence of AtGALDH acitivity on hydration degree $\mathrm{W}_{0}=\left[\mathrm{H}_{2} \mathrm{O}\right] /[\mathrm{AOT}]$ in the absence of an inhibitor has a "bell-shaped" form. In general, the size of the inner cavity of micelles where protein molecules are entrapped can be strictly controlled by varying the surfactant hydration degree $\left(\mathrm{W}_{0}\right)$, which represents the molar water-to-surfactant ratio. The optimal conditions for an enzyme functioning corresponds to the geometrical matching between the micellar inner cavity and the enzyme molecule-under these conditions the maximum of activity is observed [14]. In the case of oligomeric enzymes, the dependence of an enzyme activity on $W_{0}$ shows peaks corresponding to each oligomeric form of an enzyme [16]. There are two hydration degrees- $W_{0}=22-24$ and $W_{0}=26-28-$ at which AtGALDH activity optima are observed corresponding to monomeric and dimeric forms 
of the enzyme. It should be noted that, in aqueous solution, AtGALDH functions in a monomeric form [11].

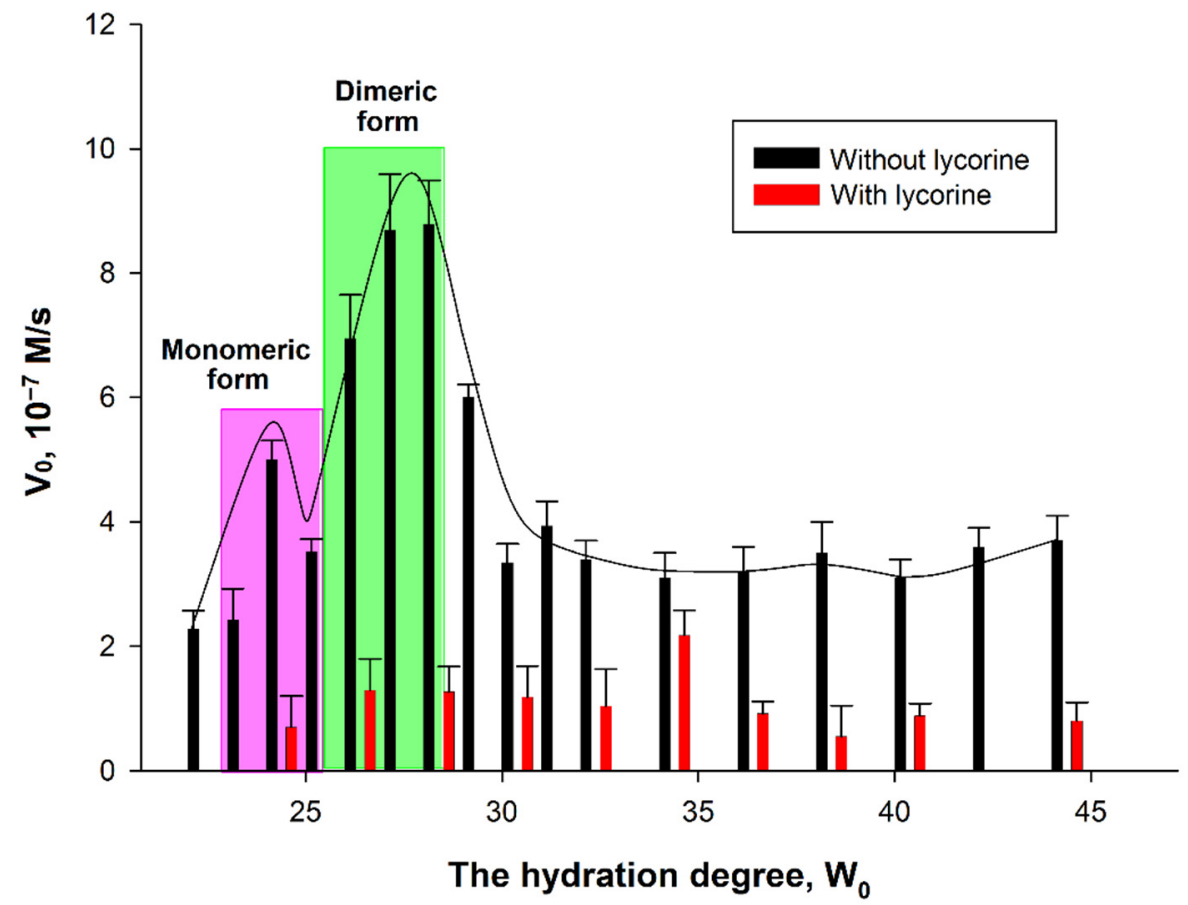

Figure 7. Dependence of AtGALDH activity on the hydration degree in the absence and presence of $800 \mu \mathrm{M}$ lycorine. The monomeric and dimeric forms of the enzymes were observed at hydration degrees $W_{0}=23-25$ and 26-28, respectively. The concentrations of AtGALDH and L-galactone1,4-lactone were $6 \times 10^{-9} \mathrm{M}$ and $1 \mathrm{mM}$, respectively. Values are presented as the mean $\pm \mathrm{SD}$ of three experiments.

It was found that the effect of inhibition by lycorine significantly depends on the hydration degree: the inhibition is more pronounced at those $W_{0}$, at which activity optima of AtGALDH are observed in the absence of lycorine (the optima corresponding to monomeric and dimeric forms are highlighted in pink and green, respectively, in Figure 7). For example, the inhibition is $86 \%$ at $\mathrm{W}_{0}=28$, corresponding to a dimeric form of AtGALDH. However, for each hydration degree, the AtGALDH activity decreases to approximately the same value $\approx 1.4 \times 10^{-7} \mathrm{M} / \mathrm{s}$ (to background level).

Statistical analysis of obtained data was carried out using the Student's $t$-test and Statistica 9.0 software (StatSoft, Tulsa, OK, USA). The differences in the AtGALDH activities in absence and presence of lycorine (values of both groups of experiments are presented in Figure 7) at all the studied hydration degrees from $W_{0}=24$ to $W_{0}=44$ were considered statistically significant, since the calculated $p$-value was less than 0.05 .

The results demonstrate that lycorine retains its inhibitory effect on AtGALDH activity in a micellar medium (membrane model) compared to an aqueous medium. Taking into account the existence of AtGALDH in aqueous media, just in monomeric form, one should compare the inhibition in aqueous solution and in micellar media at $\mathrm{W}_{0}=24$, corresponding to the monomeric form of AtGALDH. Similar percent of inhibition was observed in both cases: $87 \%$ in aqueous medium and $86 \%$ in reverse micelles system (the concentration of lycorine is $800 \mu \mathrm{M}$ in both media). At the same time, monomeric and dimeric forms in micellar media were also inhibited by lycorine to the same extent ( $86 \%$ at $800 \mu \mathrm{M}$ lycorine).

Taking this fact into account, and the homology of AtGALDH and TcGAL, one can assume a similar effect of lycorine on TcGAL activity in a micellar medium. To verify the inhibition of TcGAL by lycorine, two series of experiments were performed varying the 
concentration of lycorine at hydration degrees $\mathrm{W}_{0}=22$ and $\mathrm{W}_{0}=28$, which correspond to the activity optima of the enzyme [5]. The results are shown in Figure 8.

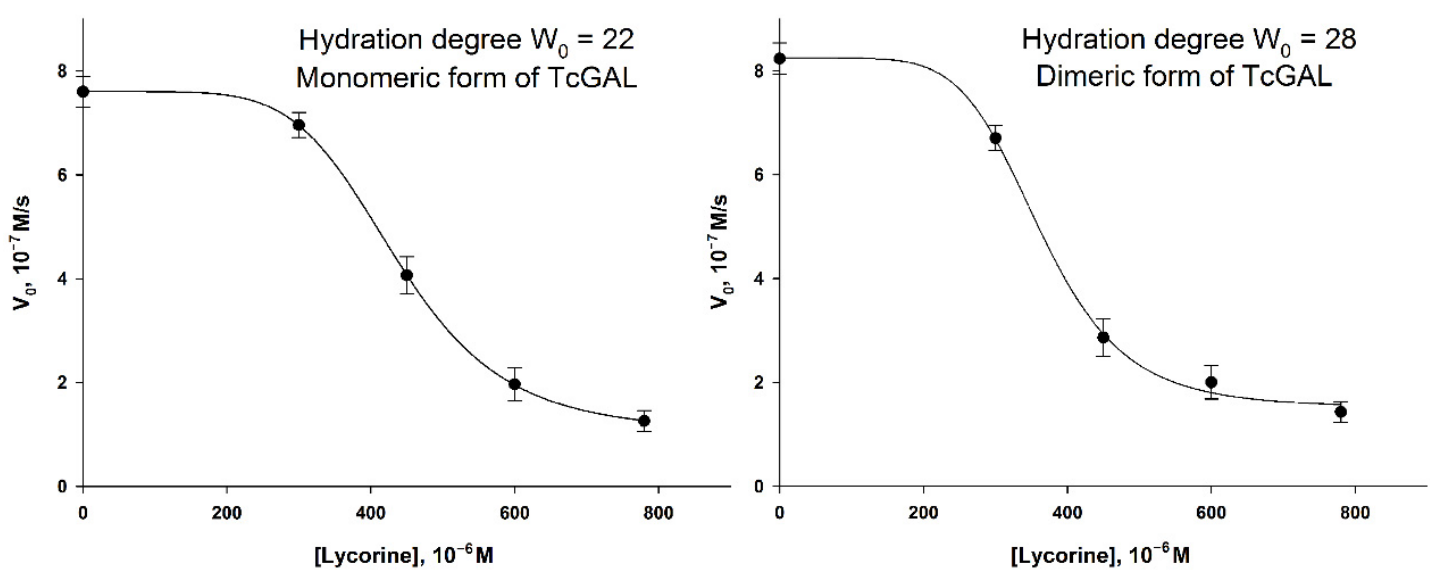

Figure 8. The inhibition of TcGAL by lycorine at hydration degrees $W_{0}=22$ and $W_{0}=28$. The concentration of TcGAL was $3.5 \times 10^{-5} \mathrm{M}$. The concentration of D-arabinono-1,4-lactone was $1 \mathrm{mM}$. Values are presented as the mean $\pm \mathrm{SD}$ of three experiments.

The "lag phase" can be observed in the lycorine concentration range of 0-300 $\mu \mathrm{M}$, which did not take place in the case of AtGALDH. The inhibition of TcGAL activity was significant only at a concentration of lycorine about $300 \mu \mathrm{M}$ and higher, while AtGALDH activity was already suppressed at $60 \mu \mathrm{M}$ of lycorine. The phenomenon cannot be explained by the distribution of lycorine between aqueous and micellar media, since there was no lag phase for AtGALDH. However, the moderate and strong inhibition effects in the AOT reverse micelles system were observed at approximately the same concentration of lycorine in the cases of both TcGAL and AtGALDH (for example 50 and 80\% inhibition, Table 5).

Table 5. The comparison of the inhibition effect of lycorine in micellar media (0,1 M AOT in n-octane, hydration degree $\mathrm{W}_{0}=22$ ). The concentrations of TcGAL and AtGALDH were $6 \times 10^{-9} \mathrm{M}$ and 3.5 $\times 10^{-5} \mathrm{M}$, correspondently. The concentrations of both substrates (D-arabinono-1,4-lactone and L-galactone-1,4-lactone) were $1 \mathrm{mM}$.

\begin{tabular}{ccc}
\hline Enzyme & Inhibition, $\%$ & Concentration of Lycorine, $\boldsymbol{\mu M}$ \\
\hline \multirow{3}{*}{ TcGAL } & 10 & 300 \\
\cline { 2 - 3 } & 50 & 450 \\
\cline { 2 - 3 } & 80 & 780 \\
\cline { 2 - 3 } AtGALDH & 10 & 60 \\
\hline & 50 & 500 \\
\hline
\end{tabular}

Thus, the effect of the inhibition of TcGAL by lycorine is rather clear, although it is not so pronounced at low concentrations of lycorine, as in the case of L-galactone-1,4-lactone dehydrogenases (including AtGALDH studied in this work); nevertheless, 50\% inhibition was observed at approximately the same concentration of lycorine $(450-500 \mu \mathrm{M})$ in both the cases of TcGAL and AtGALDH in micellar media.

The reasons of such difference in inhibition between TcGAL and AtGALDH at relatively low concentrations of lycorine $(<300 \mu \mathrm{M})$ are not clear. We proposed that TcGAL as a membrane enzyme may interact with the interface and so the active center of the enzyme is practically unavailable for lycorine. Another probable reason is that TcGAL oxidation activity is not inhibited by lycorine (AtGALDH reacts with $\mathrm{O}_{2}$ very slowly, the rate of the reaction is nearly zero). 


\section{Conclusions}

Galactonolactone oxidase from Tripanosoma cruzi can be considered as a new molecular target for the development of the drug against Tripanosoma infection. However, TcGAL is a membrane enzyme, which is insoluble and inactive in its aqueous solution, forming precipitate-inclusion bodies. The reversed micelles approach was applied here to solubilize insoluble membranotropic or membrane-bound enzymes in their functional form, thus enabling the development of the activity assay and inhibition analysis. In the case of redox enzymes, such as AtGALDH and TcGAL, this task is further complicated by the necessity to select an appropriate electron-acceptor (EA), which should be compatible with the reversed micelles complex system. For this purpose, the method for the determination of AtGALDH and TcGAL activity has been suggested, using more effective artificial electron acceptors. Being homologous, these two enzymes differ substantially in their membranotropic properties: TcGAL is insoluble in water and requires membrane, while AtGALDH functions both in aqueous and micellar media. The comparison of these enzymes is important in terms of the understanding of mechanisms of membrane enzymes actions depending on their origin (from plant or protozoa).

The method suggested is based on the micellar approach, enabling the studying of insoluble membranotropic enzymes, and aqueous media can be served as a control, using water-soluble enzymes. The optimal assay conditions are: $\mathrm{pH}$ 8.8., phenazine methosulfate is used as an EA in combination with 2,6-dichlrophenolindophenol dye (wavelength $=550 \mathrm{~nm}$ in micellar media and $600 \mathrm{~nm}$ in aqueous media).

The improved method poses some advantages in comparison with the previously described one [5], since the proposed combination of phenazine methosulfate and 2,6dichlorophenolindophenol can be applied at the $\mathrm{pH}$ optima of TcGAL and AtGALDH, and provides high ratio of the rates of the main and background reactions $\left(\mathrm{V}_{\max } / \mathrm{V}_{\text {background }}=160\right)$. Besides this, the method can be applied for some other dehydrogenases and oxidoreductases using the same or analogue EAs (for example, pyrroloquinoline quinone (PQQ)glucose dehydrogenase [28] or lipoamide dehydrogenase [16]).

We have demonstrated the possibility to use this assay for the inhibition analysis of AtGALDH and TcGAL, with the elucidation of mechanism and inhibition parameters, which otherwise could not be possible. The inhibitory effect of lycorine activity was also studied. An inhibition of $50 \%$ was observed at approximately the same concentration of lycorine $(500 \mu \mathrm{M})$, both in the case of TcGAL and AtGALDH in micellar media. The mixed competitive mechanism of the inhibition of AtGALDH by lycorine in an aqueous medium was established.

Therefore, here we demonstrated the possibilities of the new improved assay for searching for new effective inhibitors of membrane enzymes. The results obtained indicate the potential of the approach suggested for the development a high-throughput assay for a targeted screening of a chemical compound library to find a selective inhibitor for TcGAL.

Author Contributions: Conceptualization, E.V.K.; methodology, E.V.K.; validation, A.A.C.; formal analysis, A.A.C. and E.V.K.; investigation, A.A.C.; resources, E.V.K.; data curation, E.V.K.; writingoriginal draft preparation, A.A.C.; writing-review and editing, A.A.C. and E.V.K.; visualization, A.A.C.; supervision, E.V.K.; project administration, E.V.K. All authors have read and agreed to the published version of the manuscript.

Funding: This research received no external funding.

Institutional Review Board Statement: Not applicable.

Informed Consent Statement: Not applicable.

Data Availability Statement: All relevant data are provided within this manuscript.

Conflicts of Interest: The authors declare no conflict of interest. 


\section{References}

1. Sanders, C.R.; Hutchison, J.M. Membrane properties that shape the evolution of membrane enzymes. Curr. Opin. Struct. Biol. 2018, 51, 80-91. [CrossRef] [PubMed]

2. García-Huertas, P.; Cardona-Castro, N. Advances in the treatment of Chagas disease: Promising new drugs, plants and targets. Biomed. Pharmacother. 2021, 142, 112020. [CrossRef] [PubMed]

3. Santos, S.S.; de Araújo, R.V.; Giarolla, J.; Seoud, O.E.; Ferreira, E.I. Searching for drugs for Chagas disease, leishmaniasis and schistosomiasis: A review. Int. J. Antimicrob. Agents 2020, 55, 105906. [CrossRef] [PubMed]

4. Lepesheva, G.I.; Villalta, F.; Waterman, M.R. Targeting Trypanosoma cruzi Sterol 14 $\alpha$-Demethylase (CYP51), 1st ed.; Elsevier Ltd.: Amsterdam, The Netherlands, 2011; Volume 75, ISBN 9780123858634.

5. Kudryashova, E.V.; Leferink, N.G.H.; Slot, I.G.M.; Van Berkel, W.J.H. Galactonolactone oxidoreductase from Trypanosoma cruzi employs a FAD cofactor for the synthesis of vitamin C. Biochim. Biophys. Acta-Proteins Proteom. 2011, 1814, 545-552. [CrossRef]

6. Leferink, N.G.H.; Van Duijn, E.; Barendregt, A.; Heck, A.J.R.; Van Berkel, W.J.H. Galactonolactone dehydrogenase requires a redox-sensitive thiol for optimal production of vitamin C1. Plant Physiol. 2009, 150, 596-605. [CrossRef]

7. Yabuta, Y.; Yoshimura, K.; Takeda, T.; Shigeoka, S. Molecular characterization of tobacco mitochondrial L-galactono- $\gamma$-lactone dehydrogenase and its expression in Escherichia coli. Plant Cell Physiol. 2000, 41, 666-675. [CrossRef]

8. Cao, Z.; Yang, P.; Zhou, Q. Multiple biological functions and pharmacological effects of lycorine. Sci. China Chem. 2013, 56, 1382-1391. [CrossRef] [PubMed]

9. Imai, T.; Karita, S.; Shiratori, G.I.; Hattori, M.; Nunome, T.; Ôba, K.; Hirai, M. L-Galactono- $\gamma$-lactone dehydrogenase from sweet potato: Purification and cDNA sequence analysis. Plant Cell Physiol. 1998, 39, 1350-1358. [CrossRef] [PubMed]

10. Arrigoni, O.; De Gara, L.; Paciolla, C.; Evidente, A.; De Pinto, M.C.; Liso, R. Lycorine: A powerful inhibitor of L-galactono- $\gamma$ lactone dehydrogenase activity. J. Plant Physiol. 1997, 150, 362-364. [CrossRef]

11. Leferink, N.G.H.; Van Den Berg, W.A.M.; Van Berkel, W.J.H. L-Galactono- $\boldsymbol{\gamma}$-lactone dehydrogenase from Arabidopsis thaliana, a flavoprotein involved in vitamin C biosynthesis. FEBS J. 2008, 275, 713-726. [CrossRef] [PubMed]

12. Pileni, M.P.; Zemb, T.; Petit, C. Solubilization by reverse micelles: Solute localization and structure perturbation. Chem. Phys. Lett. 1985, 118, 414-420. [CrossRef]

13. Vos, K.; Laane, C.; Weijers, S.R.; Van Hoek, A.; Veeger, C.; Visser, A.J.W.G. Time-resolved fluorescence and circular dichroism of porphyrin cytochrome $\mathrm{c}$ and Zn-porphyrin cytochrome $\mathrm{c}$ incorporated in reversed micelles. Eur. J. Biochem. 1987, 169, 259-268. [CrossRef] [PubMed]

14. Kudryashova, E.V.; Bronza, V.L.; Vinogradov, A.A.; Kamyshny, A.; Magdassi, S.; Levashov, A.V. Regulation of acid phosphatase in reverse micellar system by lipids additives: Structural aspects. J. Colloid Interface Sci. 2011, 353, 490-497. [CrossRef] [PubMed]

15. Kudryashova, E.V.; Visser, A.J.W.G.; van Berkel, W.J.H. Monomer formation and function of p-Hydroxybenzoate hydroxylase in reverse micelles and dimethylsulfoxide/water mixtures. ChemBioChem 2008, 9, 413-419. [CrossRef]

16. Klyachko, N.L.; Shchedrina, V.A.; Efimov, A.V.; Kazakov, S.V.; Gazaryan, I.G.; Kristal, B.S.; Brown, A.M. pH-dependent substrate preference of pig heart lipoamide dehydrogenase varies with oligomeric state: Response to mitochondrial matrix acidification. $J$. Biol. Chem. 2005, 280, 16106-16114. [CrossRef] [PubMed]

17. Khmel'Nitskii, Y.L.; Levashov, A.V.; Klyachko, N.L.; Martinek, K. A Microheterogeneous Medium for Chemical (Enzymic) Reactions Based on a Colloidal Solution of Water in an Organic Solvent. Russ. Chem. Rev. 1984, 53, 319-331. [CrossRef]

18. Schertl, P.; Sunderhaus, S.; Klodmann, J.; Gergoff Grozeff, G.E.; Bartoli, C.G.; Braun, H.P. L-galactono-1,4-lactone dehydrogenase (GLDH) forms part of three subcomplexes of mitochondrial complex I in Arabidopsis thaliana. J. Biol. Chem. 2012, 287, 14412-14419. [CrossRef]

19. Frébortová, J.; Fraaije, M.W.; Galuszka, P.; Šebela, M.; Peč, P.; Hrbáč, J.; Novák, O.; Bilyeu, K.D.; English, J.T.; Frébort, I. Catalytic reaction of cytokinin dehydrogenase: Preference for quinones as electron acceptors. Biochem. J. 2004, 380, 121-130. [CrossRef]

20. Christ-Hazelhof, E.; Nugteren, D.H. Purification and characterisation of prostaglandin endoperoxide D-isomerase, a cytoplasmic, glutathione-requiring enzymE. Biochim. Biophys. Acta (BBA)/Lipids Lipid Metab. 1979, 572, 43-51. [CrossRef]

21. Biasutti, M.A.; Abuin, E.B.; Silber, J.J.; Correa, N.M.; Lissi, E.A. Kinetics of reactions catalyzed by enzymes in solutions of surfactants. Adv. Colloid Interface Sci. 2008, 136, 1-24. [CrossRef]

22. Liang, Y.; Yuan, X.; Zeng, G.; Zhong, H.; Li, H.; Wang, W. Effects of surfactants on enzyme-containing reversed micellar system. Sci. China Chem. 2011, 54, 715-723. [CrossRef]

23. Martinek, K.; Klyachko, N.L.; Kabanov, A.V.; Khmelnitsky, Y.L.; Levashov, A.V. Micellar enzymology: Its relation to membranology. BBA-Biomembr. 1989, 981, 161-172. [CrossRef]

24. Mu, H.M.; Wang, R.; Li, X.D.; Jiang, Y.M.; Peng, F.; Xia, B. Alkaloid accumulation in different parts and ages of Lycoris chinensis. Zeitschrift Naturforsch.-Sect. C J. Biosci. 2010, 65, 458-462. [CrossRef] [PubMed]

25. De Gara, L.; Tommasi, F. Further researches upon the inhibiting action of lycorine on ascorbic acid biosynthesis. Boll. Soc. Ital. Biol. Sper. 1990, 66, 953-960. [PubMed]

26. Evtugyn, G. Biosensors: Essentials; Springer: Berlin/Heidelberg, Germany, 2014; Volume 84, ISBN 9783642402401.

27. Lloyd, M.D. Beginner's Guide Steady-State enzyme kinetics Beginner's Guide. Biochemist 2021, 43, 40-45. [CrossRef]

28. Komkova, M.A.; Orlov, A.K.; Galushin, A.A.; Andreev, E.A.; Karyakin, A.A. Anchoring PQQ-Glucose Dehydrogenase with Electropolymerized Azines for the Most Efficient Bioelectrocatalysis. Anal. Chem. 2021, 93, 12116-12121. [CrossRef] [PubMed] 